\title{
Left ventricular diastolic function associated with common genetic variation in ATP12A in a general population
}

\author{
Judita Knez ${ }^{1,2}$, Erika Salvi ${ }^{3}$, Valérie Tikhonoff ${ }^{4,5}$, Katarzyna Stolarz-Skrzypek ${ }^{6}$, Andrew Ryabikov ${ }^{7,8}$, Lutgarde Thijs ${ }^{1}$, \\ Daniele Braga ${ }^{3}$, Malgorzata Kloch-Badelek ${ }^{6}$, Sofia Malyutina ${ }^{7,8}$, Edoardo Casiglia ${ }^{4}$, Danuta Czarnecka ${ }^{6}$, \\ Kalina Kawecka-Jaszcz ${ }^{6}$, Daniele Cusi ${ }^{3}$, Tim Nawrot ${ }^{9,10}$, Jan A Staessen ${ }^{1,11}$ and Tatiana Kuznetsova ${ }^{1 *}$
}

\begin{abstract}
Background: Left ventricular (LV) function depends on the activity of transmembrane electrolyte transporters. Failing human myocardium has lower $\mathrm{Na}^{+} / \mathrm{K}^{+}$ATPase expression and higher intracellular sodium concentrations. The ATP12A gene encodes a catalytic subunit of an ATPase that can function as a $\mathrm{Na}^{+} / \mathrm{K}^{+}$pump. We, therefore, investigated the association between LV function and common genetic variants in ATP12A.

Methods: A random sample of 1166 participants (53.7\% women; mean age 49.5 years, $44.8 \%$ hypertensive) was recruited in Belgium, Poland, Italy and Russia. We measured transmitral early and late diastolic velocities ( $E$ and $A$ ) by pulsed wave Doppler, and mitral annular velocities ( $e^{\prime}$ and $a^{\prime}$ ) by tissue Doppler. Using principal component analysis, we summarized 7 Doppler indexes - namely, $E, A, e^{\prime}$ and $a^{\prime}$ velocities, and their ratios (E/A, $e^{\prime} / a^{\prime}$, and $\left.E / e^{\prime}\right)$ - into a single diastolic score. We genotyped 5 tag SNPs (rs963984, rs9553395, rs10507337, rs12872010, rs2071490) in ATP12A. In our analysis we focused on rs10507337 because it is located within a transcription factor binding site.

Results: In the population-based analyses while adjusting for covariables and accounting for family clusters and country, rs10507337 C allele carriers had significantly higher E/A $(P=0.003), e^{\prime}\left(P=5.8 \times 10^{-5}\right), e^{\prime} / a^{\prime}(P=0.003)$ and diastolic score $(P=0.0001)$ compared to $\Pi$ homozygotes. Our findings were confirmed in the haplotype analysis and in the family-based analyses in 74 informative offspring.

Conclusions: LV diastolic function as assessed by conventional and tissue Doppler indexes including a composite diastolic score was associated with genetic variation in ATP12A. Further experimental studies are necessary to clarify the role of ATP12A in myocardial relaxation.
\end{abstract}

Keywords: Epidemiology, Echocardiography, Diastolic function, ATP12A

\section{Background}

Left ventricular (LV) diastolic dysfunction refers to a condition in which abnormalities in LV function are present during diastole and is characterized by impaired relaxation and/or filling of the heart. LV diastolic dysfunction is associated with common risk factors such as hypertension and it can progress to symptomatic heart failure [1]. The prevalence of subclinical diastolic dysfunction in the

\footnotetext{
* Correspondence: tatiana.kouznetsova@med.kuleuven.be

'KU Leuven Department of Cardiovascular Sciences, Research Unit Hypertension and Cardiovascular Epidemiology, University of Leuven, Leuven, Belgium

Full list of author information is available at the end of the article
}

general population increases with age and is as high as 25.1\% [2-5]. Echocardiographic measurement of diastolic Doppler velocities of the transmitral blood flow (E and A peaks) and the mitral annular movement (e' and a' peaks) during early and late diastole allows the non-invasive assessment of LV diastolic function [6]. Moreover, low e' velocity measured by Tissue Doppler Imaging (TDI) significantly and independently from other cardiovascular risk factors predicted higher risk of fatal and nonfatal cardiovascular events in patients with hypertension [7] or heart failure [8] and in general population [9]. Recently we demonstrated significant heritability of the diastolic Doppler indexes [10]. Understanding to what extent genetic 
factors along with anthropometric, hemodynamic factors and lifestyle influence diastolic Doppler indexes is an important issue in view of the relation of LV diastolic dysfunction with outcome.

Cardiomyocyte contraction and relaxation depend on the balance of electrolytes (calcium, sodium, potassium, etc.) across the cellular membranes $[11,12]$. The electrolyte gradients are maintained by transmembrane channels and adenosine triphosphate (ATP) dependent pumps. Experimental [13] and clinical studies [14] demonstrated that intracellular sodium concentration $\left(\mathrm{Na}^{+}\right)$is increased in failing cardiomyoctes as compared to normal myocardium. The possible cause for this observation might be related to changes in $\mathrm{Na}^{+} / \mathrm{K}^{+}$ATPase expression and/or function [11,12]. Among the genes that encode the catalytic alpha subunit of $\mathrm{Na}^{+} / \mathrm{K}^{+}$-ATPase $[15,16]$, ATP12A belongs to the family of P-type cation transport ATPases that can function as $\mathrm{Na}^{+} / \mathrm{K}^{+}$ATPase in human cells [17]. Taking together, genetic variability in ATP12A might influence myocardial $\mathrm{Na}^{+}$handling and consequently myocardial function. We recently genotyped top SNPs that might be associated with cardiovascular phenotypes and that have been identified in a recent genome-wide association study [18]. Among these SNPs common genetic variants in ATP12A were genotyped. Therefore, in the Flemish Study on Environment, Genes and Health Outcomes (FLEMENGHO) and the European Project On Genes in Hypertension (EPOGH) we investigated whether echocardiographic variables reflecting LV diastolic function are associated with common genetic variants in ATP12A.

\section{Methods}

\section{Study participants}

From August 1985 until December 2005, we randomly recruited a family-based population sample (the FLEMENGHO cohort) from a geographically defined area in northern Belgium as described in previous publications [4]. EPOGH recruited nuclear families from 1999 until 2001. The EPOGH investigators were trained at the Studies Coordinating Centre in Leuven, Belgium, and applied the same protocol, questionnaires and procedures, as used in FLEMENGHO [3]. All study participants provided a signed informed consent and the study was approved by the Ethical Committees of the University of Leuven, the University of Padova, Jagiellonian University Medical College, and the Novosibirsk Institute of Internal and Preventive Medicine. All clinical investigations were conducted according to the principles expressed in the Declaration of Helsinki. The initial response rate at enrolment was $75.0 \%$.

In the FLEMENGHO study, from May 2005 until January 2010, we invited 1055 former participants from a previously identified random population for a follow-up examination at the field centre, including echocardiography. Of those,
828 renewed their written consent. In the EPOGH study, from January 2007 until September 2009, we invited 631 former participants for an examination including echocardiography. Of those, 561 gave their consent in writing. We excluded 223 subjects from analysis, because DNA was missing or of bad quality $(n=217)$, atrial fibrillation $(n=1)$ or because LV diastolic Doppler indexes could not be reliably measured $(n=5)$. Thus, 1166 subjects were analyzed including 777 FLEMENGHO participants (Noorderkempen, Belgium) and 389 EPOGH participants from Krakow, Poland $(n=143)$, Mirano, Italy $(n=109)$ and Novosibirsk, Russia $(n=137)$.

\section{Echocardiography}

The participants refrained from smoking, heavy exercise, and drinking alcohol or caffeine-containing beverages for at least 3 hours before echocardiography.

\section{Data acquisition}

In each center one experienced physician did the ultrasound examination, using a Vivid7 Pro (GE Vingmed, Horten, Norway) interfaced with a 2.5- 3.5 MHz phasedarray probe, according to a standardized protocol as published elsewhere $[3,4]$. With the subjects in partial left decubitus and breathing normally, the observer obtained images, together with a simultaneous ECG signal, from the parasternal long and short axes and from the apical 4- and 2-chamber long-axis views. M-mode echocardiograms of the LV were recorded from the parasternal long-axis view under control of the $2 \mathrm{D}$ image. The ultrasound beam was positioned just below the mitral valve at the level of the posterior chordae tendineae. To record mitral flow velocities from the apical window, we positioned the Doppler sample volume at the mitral valve tips.

Using TDI, the observer recorded low-velocity, highintensity myocardial velocity at a high frame rate $(>190$ FPS), while adjusting the imaging angle to ensure parallel alignment of the ultrasound beam with the myocardial segment of interest. From the apical window, a $5 \mathrm{~mm}$ Doppler sample was placed at the septal, lateral, inferior and posterior sites of the mitral annulus to obtain the pulsed wave TDI velocities.

\section{Off-line analysis}

All echocardiographic recordings included at least 5 cardiac cycles and were digitally stored for off-line analysis. One experienced observer (TK) analyzed the digitally stored images from all centers, using the EchoPac software, version 4.0.4 (GE Vingmed, Horten, Norway), selecting and averaging 3 cardiac cycles obtained at the end-expiration. The observer was blinded to the genetic results. LV internal diameter and interventricular septal and posterior wall thickness were measured at end- 
diastole from the 2 dimensionally guided M-mode tracing according to the guidelines. When optimal orientation of M-mode ultrasound beam could not be obtained (in approximately $5 \%$ of echocardiographic recordings), the reader performed linear measurements on correctly oriented two-dimensional images. End-diastolic left ventricular dimensions were used to calculate LV mass by an anatomically validated formula. LV mass was indexed to body surface area (BSA). LV end-systolic and enddiastolic volumes were measured off-line using the standard biplane Simpson's method.

We assessed LV diastolic function using recordings of conventional blood flow and tissue Doppler velocities. Pulsed-wave Doppler signals of transmitral blood flow were used to measure peak early $(\mathrm{E})$ and late $(\mathrm{A})$ diastolic velocities. From the pulsed wave TDI recordings, we measured the early (e') and late (a') peak diastolic velocities of the mitral annulus displacement, and the e'/ a' ratio at the 4 acquisition sites. We calculated the E/e' ratio by dividing transmitral $E$ peak by e' averaged from the 4 acquisition sites. As reported previously [3], the inter-observer intra-session reproducibility across the four sampling sites ranged from $4.48 \%$ to $5.34 \%$ for e' velocities and from $3.96 \%$ to $4.52 \%$ for a' velocities.

\section{Other measurements}

We administered a standardized questionnaire to collect detailed information on subjects' medical history, smoking and drinking habits, and intake of medications. The conventional blood pressure was the average of five consecutive auscultatory readings obtained with the subject in the seated position. Hypertension was defined as a blood pressure of at least $140 \mathrm{~mm} \mathrm{Hg}$ systolic or $90 \mathrm{~mm} \mathrm{Hg}$ diastolic or as use of antihypertensive drugs. Body mass index was weight in kilograms divided by the square of height in meters.

\section{Genotypes}

We extracted DNA from white blood cells. For genotyping of ATP12A SNPs we used $15 \mathrm{~K}$ Illumina Infinium custom chip (Illumina Inc, San Diego, CA), designed for the HYPERGENES project [18] to cover genes deemed to be relevant for hypertension and related target organ damage. Detailed information on the procedure of tag SNP selection for the custom chip is provided in Additional file 1. The human ATP12A gene lies within chromosome 13q12.1-q12.3 (http://www.ncbi.nlm.nih.gov/gene/479) and spans approximately $32 \mathrm{~kb}$. It contains 23 exons and 22 introns [19]. The selected 8 tag SNPs were annotated according to the Genome Reference Consortium Human Build 37 hg19 (Additional file 1: Table S1). Three SNPs were excluded from the statistical analysis because of minor allele frequency $(\mathrm{MAF})=0.02$ (rs2289909), genotyping callrate $<99 \%$ (rs1867767) or bad cluster according to Illumina
Genome Studio (rs1001806, Additional file 1: Table S1). Of remaining 5 SNPs, rs963984, rs12872010 and rs2071490 lie within coding regions, but do not alter the amino acid sequence of the coded protein (synonymous). Two other SNPs (rs9553395 and rs10507337) are located in the 5' flanking region, with rs10507337 within a transcription factor binding site.

\section{Statistical methods}

For database management and statistical analysis, we used SAS software, version 9.3 (SAS Institute, Cary, $\mathrm{NC}$ ). We compared means and proportions by the $\mathrm{z}$ test and by the $X^{2}$ test, respectively. We tested minimum allele frequency, proportion of missing genotypes and Hardy-Weinberg equilibrium using JMP Genomics, version 6.1 (SAS Institute, Cary, NC). We reconstructed haplotypes using the PROC HAPLOTYPE procedure implemented in the genetics module of the SAS software.

\section{Principal component analysis}

The diastolic Doppler velocities are measured using similar technique (Doppler) and are highly intra-correlated. Therefore, some redundancy in the cumulative variance of the Doppler velocities exists. This redundancy can be decreased by a mathematical procedure, the principal component analysis. This thechnique enables summarization of intra-correlated variables into an artificial score that carries information from all the contributing variables. Individual values of the artificial score are calculated by summarization of optimally weighted values of the contributing measured indexes. We, therefore, summarized transmitral $\mathrm{E}$ and A blood flow velocities, averaged TDI e' and a' mitral annulus velocities and their ratios (E/A, e'/a', E/e') into a single composite diastolic score. Normality of diastolic score distribution was evaluated by ShapiroWilk's statistic and skewness by computation of the coefficient of skewness. The association between the composite diastolic score and velocities was described using factors loadings. We performed stepwise linear regression to identify correlates of the composite diastolic score with sex, age, height, weight, body mass index, heart rate, systolic and diastolic blood pressures, left ventricular mass index, and ejection fraction. We set the $P$ value for variables to enter and stay in the regression models at 0.10 .

\section{Population-based analysis}

First, we performed the association analyses of dependent variables (LV phenotypes) with the genotypes or haplotypes of interest by use of a mixed model in each examination center. Covariables with known relevance for LV structure and function [3] were included in the models as fixed effects, while family clusters was modeled as a random effect. Then we performed the association analyses in all centers combined while examination centers were modeled as 
random effects. We tested whether observed effect sizes are homogeneous across examination centers. The heterogeneity test statistic (Cochran's Q) was computed by summing the squared deviations of each center estimate from the overall meta-analytic estimate, weighting each center contribution in the same manner as in the meta-analysis. We also reported the $P$-values corrected for multiple testing using the Bonferroni method.

\section{Family-based analysis}

We performed the transmission disequilibrium test for quantitative traits (QTDT). We evaluated the within- and between-family components of phenotypic variance using the orthogonal model as implemented by Abecasis et al.
[20] in the QTDT software (version 2.6.1; http://www.sph. umich.edu/csg/abecasis/QTDT). In this model the between-family component is sensitive to population structure whereas the within-family component is significant in the presence of transmission disequilibrium.

\section{Results}

Characteristics of participants by center, ATP12A alleles and genotypes and haplotypes frequencies

Table 1 summarizes baseline characteristics of participants by examination center. Overall, the 1166 white European participants included 626 (53.7\%) women and 522 (44.8\%) hypertensive patients of whom 315 (27.0\%) were on antihypertensive drug treatment. Mean age $( \pm \mathrm{SD})$ was $49.5 \pm$

Table 1 Characteristics of participants by country

\begin{tabular}{|c|c|c|c|c|}
\hline & Belgium & Poland & Italy & Russia \\
\hline Characteristic & $(n=777)$ & $(n=143)$ & $(n=109)$ & $(n=137)$ \\
\hline \multicolumn{5}{|l|}{ Anthropometrics } \\
\hline Female sex, $n(\%)$ & $399(51.4)$ & 78 (54.6) & $60(55.1)$ & $89(65.9)^{*}$ \\
\hline Age (years) & $51.0 \pm 15.5$ & $42.9 \pm 13.8^{*}$ & $50.3 \pm 13.5+$ & $47.7 \pm 14.5^{*} \dagger$ \\
\hline Height (cm) & $168.7 \pm 9.4$ & $169.5 \pm 9.1$ & $167.0 \pm 9.0 \dagger$ & $167.1 \pm 9.6+$ \\
\hline Weight (kg) & $75.5 \pm 14.4$ & $78.1 \pm 15.1$ & $75.2 \pm 14.2$ & $77.3 \pm 16.2$ \\
\hline Body mass index $\left(\mathrm{kg} / \mathrm{m}^{2}\right)$ & $26.5 \pm 4.3$ & $27.2 \pm 5.3$ & $26.9 \pm 4.6$ & $27.7 \pm 5.6^{*}$ \\
\hline Systolic pressure (mmHg) & $129.3 \pm 17.6$ & $137.7 \pm 18.9^{*}$ & $125.4 \pm 17.1^{*} \dagger$ & $132.4 \pm 23.8 \neq$ \\
\hline Diastolic pressure (mmHg) & $79.6 \pm 9.4$ & $84.2 \pm 11.7^{*}$ & $82.3 \pm 10.1^{*}$ & $84.4 \pm 14.1^{*}$ \\
\hline Heart rate (beats/minute) & $61.0 \pm 9.7$ & $66.4 \pm 11.2^{*}$ & $65.9 \pm 10.4^{*}$ & $66.2 \pm 10.3^{*}$ \\
\hline \multicolumn{5}{|l|}{ Questionnaire data } \\
\hline Current smoking, $n(\%)$ & $161(20.7)$ & $31(21.7)$ & $18(16.5)$ & $35(25.6)$ \\
\hline Drinking alcohol, $n$ (\%) & $309(39.8)$ & $17(11.9)^{*}$ & $53(48.6) \dagger$ & $38(27.7) \dagger \neq$ \\
\hline Hypertensive, $n(\%)$ & $323(41.6)$ & $81(56.6)^{*}$ & $51(46.8)$ & $67(48.9)$ \\
\hline Treated for hypertension, $n$ (\%) & $197(25.4)$ & $44(32.8)$ & $25(22.9)$ & $49(35.8)^{*} \ddagger$ \\
\hline \multicolumn{5}{|l|}{ Conventional echocardiography } \\
\hline LV internal diameter (cm) & $5.04 \pm 0.47$ & $5.06 \pm 0.47$ & $4.91 \pm 0.44^{*} \dagger$ & $4.99 \pm 0.41$ \\
\hline Interventricular septum (cm) & $0.98 \pm 0.17$ & $0.96 \pm 0.15$ & $0.94 \pm 0.15^{*}$ & $0.98 \pm 0.18$ \\
\hline Posterior wall (cm) & $0.90 \pm 0.14$ & $0.90 \pm 0.13$ & $0.88 \pm 0.13$ & $0.90 \pm 0.13$ \\
\hline LV mass index $\left(\mathrm{g} / \mathrm{m}^{2}\right)$ & $92.4 \pm 22.0$ & $90.8 \pm 20.9$ & $86.0 \pm 18.6^{*}$ & $91.2 \pm 21.9$ \\
\hline Ejection fraction (\%) & $63.3 \pm 6.75$ & $63.9 \pm 6.56$ & $62.6 \pm 6.15$ & $64.1 \pm 6.13$ \\
\hline \multicolumn{5}{|l|}{ Diastolic function } \\
\hline Transmitral E peak (cm/s) & $75.5 \pm 16.1$ & $74.5 \pm 14.9$ & $68.8 \pm 14.9^{*}+$ & $67.0 \pm 14.5^{*} \dagger$ \\
\hline Transmitral A peak (cm/s) & $65.0 \pm 17.4$ & $58.6 \pm 14.7^{*}$ & $59.6 \pm 19.5^{*}$ & $61.0 \pm 18.0^{*}$ \\
\hline Transmitral E/A ratio & $1.26 \pm 0.48$ & $1.36 \pm 0.44^{*}$ & $1.28 \pm 0.49$ & $1.21 \pm 0.49 \dagger$ \\
\hline TDI e' peak $(\mathrm{cm} / \mathrm{s})$ & $11.4 \pm 3.65$ & $11.3 \pm 3.51$ & $10.3 \pm 3.15^{*}+$ & $10.4 \pm 3.40^{*}$ \\
\hline TDI a' peak $(\mathrm{cm} / \mathrm{s})$ & $10.1 \pm 2.11$ & $9.08 \pm 1.93^{*}$ & $10.2 \pm 2.12 \dagger$ & $9.33 \pm 2.05^{*} \neq$ \\
\hline$e^{\prime} / a^{\prime}$ ratio & $1.26 \pm 0.71$ & $1.36 \pm 0.64$ & $1.07 \pm 0.52^{*}+$ & $1.29 \pm 0.68 \neq$ \\
\hline E/e' ratio & $7.10 \pm 2.19$ & $7.10 \pm 2.16$ & $7.15 \pm 2.26$ & $7.04 \pm 2.63$ \\
\hline Composite diastolic score & $-0.028 \pm 2.11$ & $0.41 \pm 1.93^{*}$ & $-0.27 \pm 1.92 \dagger$ & $-0.081 \pm 2.07 \dagger$ \\
\hline
\end{tabular}

Values are mean \pm SD or number of subjects (\%). LA, LV and TDI indicate left atrium, left ventricle and Tissue Doppler Imaging. Significance for between-country differences: $* P \leq 0.05$ vs Belgium; $+P \leq 0.05$ vs Poland; $\neq P \leq 0.05$ vs Italy. 
15.2 years. Polish participants were younger, had higher systolic blood pressure and less frequently reported a daily alcohol consumption of $\geq 5$ gram than participants from other centers (Table 1). Composite diastolic Doppler score were highest in the Polish center compared to the remaining centers (Table 1). Additional file 1: Table S2 presents baseline characteristics of participants by sex.

Additional file 1: Table S1 lists the chromosome position, location type, allele frequencies, call frequencies and tagged SNPs of the 8 selected ATP12A SNPs. In our analyses we focused on rs10507337 because it is located within a transcription factor binding site and, therefore, it might influence the ATP12A gene expression. The $\mathrm{r}^{2}$ reflecting linkage disequilibrium between rs10507337 and rs 12872010 was 0.83 . On the other hand, we observed no correlation between rs10507337 and three other ATP12A SNPs, rs963984, rs9553395 and rs2071490, with the $\mathrm{r}^{2}$ values ranged from 0.008 to 0.245 , respectively. The genotype frequencies of rs10507337 complied with HardyWeinberg equilibrium $(P=0.97)$. Center specific and cumulative rs10507337 genotype distributions are listed in Table 2. As there were only 5 (0.4\%) rs10507337 C allele homozygotes (all from the Belgian center), we contrasted rs10507337 C allele carriers with TT homozygotes and, therefore, use a recessive association model. Additional file 1: Table S3 lists the clinical and echocardiographic characteristics of the participants by rs10507337 genotypes. In unadjusted analysis, rs10507337 C allele carriers had lower heart rate, higher transmitral E, E/A ratio, TDI e' and e'/a' ratio compared to TT homozygotes. There were no other differences in clinical or echocardiographic parameters between these two groups.

We used the selected 5 tag SNPs of ATP12A (rs9553395, rs10507337, rs2071490, rs12872010 and rs963984; Additional file 1: Table S1) to reconstruct haplotypes. Haplotype frequencies for TTTCC, TTCCA, TTCCC, CTTCC, CTCCA, CCTTC were 67.4\%, 7.1\%, 8.5\%, 6.9\%, $2.3 \%$ and $5.3 \%$, respectively.

\section{Composite diastolic score}

Because of the high intra-correlation of Doppler diastolic velocities, we summarized these traits into a single diastolic score using principal component analysis. The first principal component accounted for $61.5 \%$ of the overall

Table 2 rs 10507337 genotype distribution by country

\begin{tabular}{llll}
\hline \multirow{2}{*}{ Country } & \multicolumn{2}{l}{ Genotype } & \\
\cline { 2 - 4 } & TT N (\%) & TC N (\%) & CC N (\%) \\
\hline Belgium $(n=777)$ & $663(85.3)$ & $109(14.0)$ & $5(0.64)$ \\
Italy $(n=109)$ & $101(92.7)$ & $8(7.34)$ & $/$ \\
Poland $(n=143)$ & $123(86.0)$ & $20(14.0)$ & $/$ \\
Russia $(n=137)$ & $130(94.9)$ & $7(5.11)$ & $/$ \\
All participants $(n=1166)$ & $1017(87.2)$ & $144(12.4)$ & $5(0.43)$ \\
\hline
\end{tabular}

variance in the 7 contributing Doppler indexes. Therefore, we used the first principal component as a normally distributed summary score (mean 0; standard deviation 1; Additional file 1: Figure S2). Figure 1 shows factor loadings for each of the diastolic Doppler measures which we used to calculate the composite diastolic score. Participants with better diastolic function profile (higher transmitral and tissue Doppler velocities during early diastole and lower E/e') had higher values of the composite score. In stepwise multiple regression, the composite diastolic score significantly and independently decreased with age, body mass index, heart rate, diastolic blood pressure and left ventricular mass index (Table 3 ). The explained variance totaled $79.2 \%$.

\section{Population-based association study}

We adjusted the model for important covariables such as sex, age, body mass index, heart rate and diastolic blood pressure as well as for non-independence of observations within families and centers. Table 4 lists the adjusted Doppler diastolic indexes and composite score by rs10507337 genotype by country and in all centers combined. When all centers were pooled (Table 4), subjects carrying at least one rs10507337 $\mathrm{C}$ allele had significantly higher values of TDI e' $\left(P=5.8 \times 10^{-5}\right)$, transmitral E/A $(P=0.003)$, TDI e'/a' $(P=0.003)$ and composite diastolic score $(P=0.0001)$. The heterogeneity tests $(P=0.04)$ suggested that there was borderline but significant heterogeneity among the centers for peaks $\mathrm{E}$ and e', e'/a' ratio and composite diastolic score (Table 4).

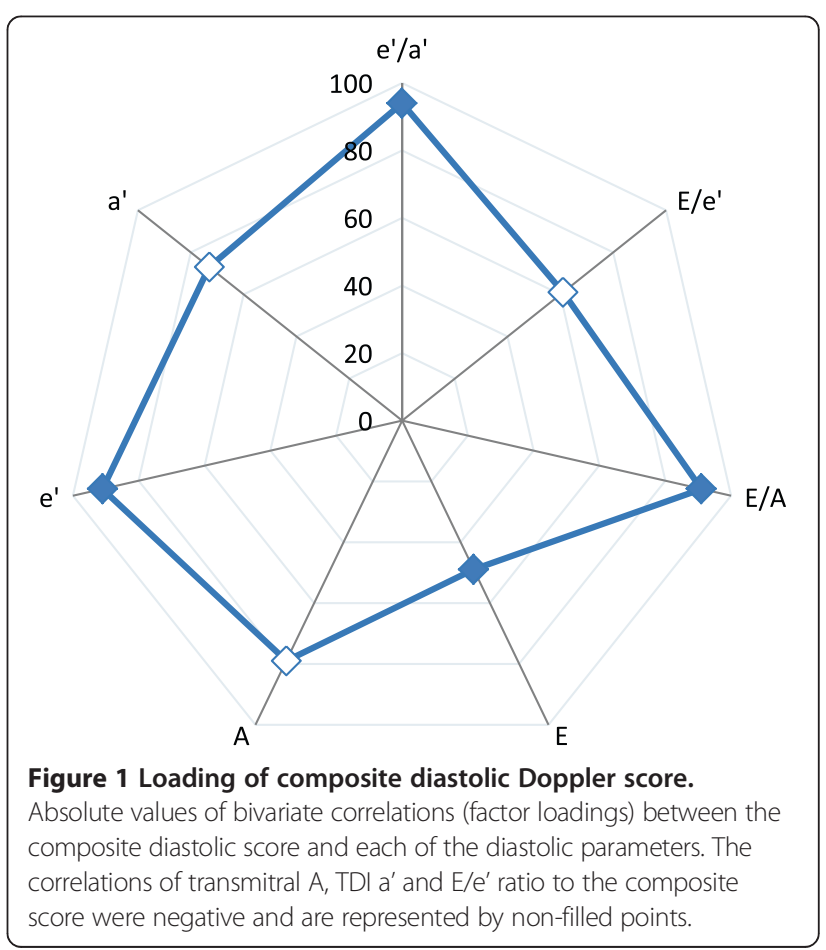


Table 3 Correlates of the composite diastolic Doppler score selected in stepwise regression

\begin{tabular}{|c|c|c|c|c|}
\hline \multirow[t]{2}{*}{ Parameter } & \multicolumn{4}{|c|}{ Diastolic Doppler score } \\
\hline & Partial $\mathrm{R}^{2}(\%)$ & Parameter estimate $\pm \mathrm{SE}$ & $95 \% \mathrm{Cl}$ & $P$-value \\
\hline Age (+10 years) & 66.0 & $-1.01 \pm 0.021$ & -1.06 to -0.98 & $<0.0001$ \\
\hline Body mass index $\left(+1 \mathrm{~kg} / \mathrm{m}^{2}\right)$ & 4.37 & $-0.080 \pm 0.0068$ & -0.093 to -0.066 & $<0.0001$ \\
\hline Heart rate (+10 beats/minute) & 7.49 & $-0.47 \pm 0.029$ & -0.52 to -0.40 & $<0.0001$ \\
\hline Diastolic pressure (+10 mmHg) & 1.08 & $-0.21 \pm 0.029$ & -0.26 to -0.15 & $<0.0001$ \\
\hline LVMI $\left(+10 \mathrm{~g} / \mathrm{m}^{2}\right)$ & 0.18 & $-0.048 \pm 0.015$ & -0.077 to -0.018 & 0.0016 \\
\hline Total R ${ }^{2}(\%)$ & 79.2 & & & \\
\hline
\end{tabular}

Values are mutually adjusted partial regression coefficients $\pm \mathrm{SE}$. Cl, confidence interval; LVMI, left ventricle mass index.

After excluding from the analyses the Polish participants, who were younger than the participants from other centers, we did not observe a heterogeneity among the remaining centers $(P>0.40)$. Haplotype analysis further confirmed that in all subjects combined, composite diastolic score $(P=0.001$; Table 5$)$ and related Doppler parameters such as TDI e', E/A, e'/a' $(P \leq 0.009$; Table 6) were significantly higher in CCTTC carriers than in noncarriers. This is the only haplotype that included rs10507337 C allele.
Our findings remained consistent after exclusion of subjects with history of coronary heart disease or valve abnormalities ( $n=67$; Additional file 1: Table S4) or subjects on antihypertensive drugs $(\mathrm{n}=315$; Additional file 1 : Table S5). For other LV phenotypes (Additional file 1: Table S6), none of the phenotype-genotype associations reached statistical significance. With adjustment for multiple testing applied, we observed that TDI e', e'/a' ratio and composite diastolic score were significantly $(P \leq 0.008)$ higher in carriers of rs12872010 $\mathrm{T}$ allele

Table 4 Adjusted Doppler diastolic indexes and composite score by rs10507337 by country and all centers combined

\begin{tabular}{|c|c|c|c|c|c|c|c|c|c|}
\hline \multirow[t]{2}{*}{ Country } & \multirow[t]{2}{*}{ Genotype } & \multicolumn{8}{|c|}{ LV diastolic indexes adjusted means \pm SE } \\
\hline & & E peak $(\mathrm{cm} / \mathrm{s})$ & A peak $(\mathrm{cm} / \mathrm{s})$ & E/A ratio & TDI e' (cm/s) & TDI a' (cm/s) & $e^{\prime} / a^{\prime}$ ratio & E/e' ratio & $\begin{array}{l}\text { Composite } \\
\text { diastolic score }\end{array}$ \\
\hline \multirow[t]{3}{*}{ Belgium $(n=777)$} & $\Pi(n=663)$ & $75.2 \pm 0.58$ & $65.3 \pm 0.51$ & $1.25 \pm 0.013$ & $11.3 \pm 0.092$ & $10.2 \pm 0.084$ & $1.23 \pm 0.018$ & $7.13 \pm 0.064$ & $-0.097 \pm 0.046$ \\
\hline & $\begin{array}{l}\text { Callele carriers } \\
(n=114)\end{array}$ & $76.9 \pm 1.32$ & $62.9 \pm 1.12$ & $1.34 \pm 0.028$ & $12.2 \pm 0.19$ & $9.99 \pm 0.16$ & $1.38 \pm 0.038$ & $6.87 \pm 0.16$ & $0.35 \pm 0.094$ \\
\hline & P & 0.22 & 0.044 & 0.0008 & $8.2 \times 10^{-6}$ & 0.15 & 0.0002 & 0.12 & $3.5 \times 10^{-6}$ \\
\hline \multirow[t]{3}{*}{ Italy $(n=109)$} & $\Pi(n=101)$ & $67.7 \pm 1.68$ & $59.3 \pm 1.32$ & $1.27 \pm 0.030$ & $10.3 \pm 0.19$ & $10.2 \pm 0.17$ & $1.05 \pm 0.035$ & $7.11 \pm 0.20$ & $-0.30 \pm 0.10$ \\
\hline & $\begin{array}{l}\text { C allele carriers } \\
(n=8)\end{array}$ & $81.2 \pm 4.80$ & $59.1 \pm 4.74$ & $1.38 \pm 0.11$ & $11.6 \pm 0.62$ & $9.87 \pm 0.58$ & $1.18 \pm 0.11$ & $7.30 \pm 0.66$ & $0.26 \pm 0.34$ \\
\hline & P & 0.006 & 0.97 & 0.34 & 0.042 & 0.58 & 0.26 & 0.78 & 0.12 \\
\hline \multirow[t]{3}{*}{ Poland $(n=143)$} & $\Pi \mathrm{T}(n=123)$ & $74.4 \pm 1.30$ & $58.4 \pm 1.06$ & $1.36 \pm 0.025$ & $11.4 \pm 0.20$ & $9.13 \pm 0.15$ & $1.37 \pm 0.030$ & $7.05 \pm 0.18$ & $0.43 \pm 0.087$ \\
\hline & $\begin{array}{l}\text { C allele carriers } \\
(n=20)\end{array}$ & $72.0 \pm 3.31$ & $58.0 \pm 2.60$ & $1.34 \pm 0.066$ & $10.8 \pm 0.51$ & $9.11 \pm 0.38$ & $1.24 \pm 0.080$ & $7.16 \pm 0.43$ & $0.17 \pm 0.22$ \\
\hline & P & 0.50 & 0.87 & 0.80 & 0.27 & 0.95 & 0.14 & 0.80 & 0.27 \\
\hline \multirow[t]{3}{*}{ Russia ( $n=137)$} & $\begin{array}{l}\Pi \pi \\
(n=130)\end{array}$ & $65.6 \pm 1.24$ & $59.8 \pm 1.03$ & $1.20 \pm 0.03$ & $10.3 \pm 0.19$ & $9.45 \pm 0.15$ & $1.24 \pm 0.044$ & $6.96 \pm 0.20$ & $-0.14 \pm 0.11$ \\
\hline & $\begin{array}{l}\text { C allele carriers } \\
(n=7)\end{array}$ & $61.8 \pm 4.86$ & $59.1 \pm 3.92$ & $1.22 \pm 0.12$ & $10.4 \pm 0.69$ & $9.74 \pm 0.57$ & $1.32 \pm 0.16$ & $6.20 \pm 0.78$ & $0.012 \pm 0.38$ \\
\hline & P & 0.45 & 0.86 & 0.84 & 0.93 & 0.61 & 0.62 & 0.34 & 0.70 \\
\hline \multirow{5}{*}{$\begin{array}{l}\text { All participants } \\
(n=1166)\end{array}$} & $\Pi(n=1017)$ & $70.9 \pm 1.74$ & $60.8 \pm 1.50$ & $1.27 \pm 0.016$ & $10.68 \pm 0.25$ & $9.66 \pm 0.24$ & $1.22 \pm 0.028$ & $7.15 \pm 0.11$ & $-0.050 \pm 0.036$ \\
\hline & $\begin{array}{l}\text { C allele carriers } \\
(n=149)\end{array}$ & $72.9 \pm 2.06$ & $59.4 \pm 1.76$ & $1.34 \pm 0.028$ & $11.36 \pm 0.29$ & $9.52 \pm 0.27$ & $1.33 \pm 0.042$ & $6.98 \pm 0.18$ & $0.28 \pm 0.083$ \\
\hline & P & 0.11 & 0.19 & 0.003 & $5.8 \times 10^{-5}$ & 0.32 & 0.003 & 0.28 & 0.0001 \\
\hline & $p^{*}$ & / & / & 0.014 & 0.0007 & / & 0.007 & / & 0.0008 \\
\hline & $P$ for heterogeneity & 0.04 & 0.81 & 0.48 & 0.04 & 0.79 & 0.04 & 0.66 & 0.04 \\
\hline
\end{tabular}

Values are least square means \pm SE adjusted for family clusters, country (in combined analysis), sex, age, body mass index, diastolic blood pressure and heart rate. TDI indicates Tissue Doppler Imaging, SE, standard error. $P$-values are for the differences between rs 10507337 T homozygotes and $C$ allele carriers. $P^{*}$-values are for the differences after Bonferroni adjustment for multiple comparisons. 
Table 5 Composite diastolic Doppler score by ATP12A haplotypes in all centers combined

\begin{tabular}{|c|c|c|c|c|c|}
\hline \multirow[b]{2}{*}{ Haplotype } & \multicolumn{4}{|c|}{ Number of coded haplotypes } & \multirow[b]{2}{*}{$P^{*}$} \\
\hline & 00 & 01 & 11 & $P$ & \\
\hline \multicolumn{6}{|l|}{ TTTCC } \\
\hline N (\%) & $104(9.0)$ & $552(47.3)$ & $510(43.7)$ & & \\
\hline Adjusted & $0.19 \pm 0.10$ & $-0.012 \pm 0.045$ & $-0.057 \pm 0.048$ & 0.07 & / \\
\hline \multicolumn{6}{|l|}{ TTCCA } \\
\hline N (\%) & $1005(86.2)$ & $157(13.5)$ & $4(0.34)$ & & \\
\hline Adjusted & $-0.020 \pm 0.037$ & $0.042 \pm 0.078$ & & 0.45 & / \\
\hline \multicolumn{6}{|l|}{ TTCCC } \\
\hline N (\%) & $972(83.4)$ & $184(15.8)$ & $10(0.86)$ & & \\
\hline Adjusted & $0.005 \pm 0.037$ & $-0.080 \pm 0.072$ & & 0.27 & / \\
\hline \multicolumn{6}{|l|}{ CTTCC } \\
\hline N (\%) & $1009(86.5)$ & $154(13.2)$ & $3(0.26)$ & & \\
\hline Adjusted & $-0.019 \pm 0.038$ & $0.041 \pm 0.079$ & & 0.47 & / \\
\hline \multicolumn{6}{|l|}{ CTCCA } \\
\hline No. (\%) & $1113(95.5)$ & $53(4.55)$ & / & & \\
\hline Adjusted & $-0.005 \pm 0.035$ & $-0.12 \pm 0.13$ & & 0.41 & / \\
\hline \multicolumn{6}{|l|}{ CCTTC } \\
\hline No. (\%) & $1047(89.8)$ & $115(9.86)$ & $4(0.34)$ & & \\
\hline Adjusted & $-0.039 \pm 0.036$ & $0.27 \pm 0.092$ & & 0.001 & 0.003 \\
\hline
\end{tabular}

Values are least square means \pm SE adjusted for family clusters, country, sex, age, body mass index, diastolic blood pressure and heart rate. $P^{*}$-values are for the differences after Bonferroni adjustment for multiple comparisons.

compared to non-carriers (Additional file 1: Table S7). These associations were somewhat expected given the high correlation between ATP12A rs10507337 and rs12872010. We did not observe any significant associations with other tested SNPs (Additional file 1: Tables S8, S9 and S10) or haplotypes (Table 5).

\section{Family-based association study}

Our study population $(n=1166)$ included 749 founders and 417 offspring from 357 families. In our family-based

Table 6 Adjusted Doppler diastolic indexes and composite score by CCTTC haplotype in all centers combined

\begin{tabular}{|c|c|c|c|}
\hline \multirow[b]{2}{*}{ LV diastolic indexes } & \multicolumn{2}{|l|}{ CCTTC } & \multirow[t]{2}{*}{$P$} \\
\hline & $00(n=1047)$ & 01 or $11(n=119)$ & \\
\hline Transmitral E peak (cm/s) & $71.0 \pm 1.75$ & $72.3 \pm 2.15$ & 0.33 \\
\hline Transmitral A peak $(\mathrm{cm} / \mathrm{s})$ & $60.7 \pm 1.50$ & $59.5 \pm 1.83$ & 0.26 \\
\hline Transmitral E/A ratio & $1.27 \pm 0.016$ & $1.34 \pm 0.031$ & 0.009 \\
\hline TDI e' peak (cm/s) & $10.7 \pm 0.25$ & $11.3 \pm 0.30$ & 0.0006 \\
\hline TDI a' peak (cm/s) & $9.65 \pm 0.24$ & $9.53 \pm 0.28$ & 0.42 \\
\hline$e^{\prime} / a^{\prime}$ ratio & $1.23 \pm 0.028$ & $1.33 \pm 0.045$ & 0.008 \\
\hline E/e' ratio & $7.15 \pm 0.11$ & $6.97 \pm 0.19$ & 0.29 \\
\hline Composite diastolic score & $-0.039 \pm 0.036$ & $0.27 \pm 0.092$ & 0.001 \\
\hline
\end{tabular}

Values are least square means \pm SE adjusted for family clusters, country, sex, age, body mass index, diastolic blood pressure and heart rate. $P$-values are for the differences between CCTTC carriers and the rest of ATP12A genotypes. analyses we used 74 informative offspring belonging to 35 families (mean age $34.6 \pm 8.2$ years; $56.9 \%$ women). The number of offspring per informative family amounted to one in 10 families, two in 17 families, three in 4 families, and more than three in 4 families. We adjusted the QTDT analyses as described in the previous section. For LV diastolic function indexes in relation to rs10507337 genotypes, the orthogonal model did not demonstrate population stratification $(P \geq 0.08)$. Transmitral E/A, TDI e', TDI e'/a' and composite diastolic score significantly increased with transmission of at least one $\mathrm{C}$ allele to offspring (Table 7). The effect sizes of the withinfamily components averaged $0.17\left(\chi^{2}=6.61, P=0.010\right)$ for E/A, $1.19 \mathrm{~cm} / \mathrm{s}\left(\chi^{2}=8.61, P=0.003\right)$ for e', $0.26\left(\chi^{2}=9.13\right.$, $P=0.003)$ for $\mathrm{e}^{\prime} / \mathrm{a}^{\prime}$ and $0.62\left(\mathrm{X}^{2}=9.04, P=0.003\right)$ for composite score (Table 7).

\section{Discussion}

The main finding of the present study was that LV diastolic function as assessed by conventional and tissue Doppler indexes was associated with genetic variation in the ATP12A promoter. Carriers of rs10507337 C allele had better pattern of myocardial relaxation as compared to non-carriers. Because rs10507337 $\mathrm{C}$ allele occurred only in one reconstructed haplotype (CCTTC), we found also the significant associations between this haplotype and LV diastolic function indexes. The family-based analyses 
Table 7 Results of the QTDT analyses for the rs 10507337 $\mathrm{C}$ allele in relation to Doppler diastolic indexes and composite score

\begin{tabular}{llll}
\hline & Parameter estimate & $\mathbf{x}^{\mathbf{2}}$ & $\boldsymbol{P}$ \\
LV diastolic indexes & & & \\
\hline Transmitral E peak (cm/s) & 1.40 & 0.22 & 0.64 \\
Transmitral A peak (cm/s) & -3.30 & 1.59 & 0.21 \\
Transmitral E/A ratio & 0.17 & 6.61 & 0.010 \\
TDl e' peak (cm/s) & 1.19 & 8.61 & 0.003 \\
TDI a' peak (cm/s) & -0.25 & 0.21 & 0.65 \\
TDI e'/a' ratio & 0.26 & 9.13 & 0.003 \\
E/e' ratio & -0.33 & 0.74 & 0.39 \\
Composite diastolic score & 0.62 & 9.04 & 0.003 \\
\hline
\end{tabular}

The orthogonal model accounted for between- and within-family variability components. The parameter estimate for the within-family component of the rs10507337 C allele indicates the direction and size of the association. Analyses were adjusted for center, sex, age, body mass index, heart rate and diastolic blood pressure. TDI, Tissue Doppler Imaging.

included only 74 informative offspring but nevertheless confirmed that transmission of at least one $\mathrm{C}$ allele to offspring was associated with higher transmitral and tissue Doppler velocities during early diastole and composite diastolic score. On the contrary, we did not observe any association of the genetic variants in ATP12A and other LV phenotypes including LV mass index and ejection fraction.

The gold standard for assessing diastolic function remains the pressure-volume relationship, but this requires an invasive approach. Doppler measurements of mitral inflow and the TDI technique open up the possibility of evaluating non-invasively diastolic function [6]. In our study, we assessed LV diastolic function non-invasively using the transmitral flow and the TDI mitral annular velocities. Previous studies validated these indexes versus invasive measures of diastolic function [6]. LV diastolic dysfunction is defined as functional abnormalities that exist during LV relaxation and filling. Impaired myocardial relaxation is characterized by decreased early (E peak), but enhanced atrial LV filling (A peak) as well as less vigorous mitral annulus motion during early diastole (TDI e' peak). Moreover, e' peak velocity along the LV longitudinal axis is less susceptible to the effects of an increased preload and therefore provides a more direct measure of myocardial relaxation than, for instance, the transmitral E peak velocity. In addition, combining transmitral flow velocity with annular velocity (E/e' ratio) might be a tool for assessing the LV filling pressure, which combines the influence of the transmitral driving pressure and myocardial relaxation [6].

To our knowledge, no previous study reported the association of cardiovascular phenotypes with the ATP12A gene. So far, only a whole DNA array survey in spontaneously hypertensive rats identified the ATP12A gene among probable candidate genes for hypertension [21]. Kinoshita et al. [21] demonstrated that lower ATP12A expression might contribute to development of hypertension in spontaneously hypertensive rats. There is a high level of structural similarity (up to 86\%) in the ATP $12 A$ gene between humans and rodents [16]. The ATP12A gene product is a catalytic alpha (ATP12A $\alpha$ ) subunit of an ATP dependent transmembrane electrolyte pump family $\left(\mathrm{X}^{+}-\mathrm{K}^{+}\right.$-ATPases) $[16,19]$ which is expressed in human myocardium (http://www.genecards.org/cgi-bin/ carddisp.pl?gene $=$ ATP12A). Functional $\mathrm{X}^{+}-\mathrm{K}^{+}$-ATPases are heterodimers, composed of alpha and beta subunits. Alpha subunits of ATPases have catalytic and ionbinding properties [22]. Previous studies in Xenopus oocytes and human HEK293 cells demonstrated that ATP12A $\alpha$ could be assembled to ATPase that functions as a $\mathrm{Na}^{+}-\mathrm{K}^{+}$-pump $[17,23]$. Moreover, ATP12A $\alpha$ is sensitive to cardiac glycoside ouabain, specific inhibitor of $\mathrm{Na}^{+}-\mathrm{K}^{+}$-ATPases [24].

Small perturbations in $\mathrm{Na}^{+}$concentration due to changes of $\mathrm{Na}^{+}-\mathrm{K}^{+}$-ATPase expression and activity, might lead to important changes of $\mathrm{Ca}^{2+}$ extrusion, a process central for myocardial contractility and relaxation [11,12]. Schwinger et al. $[25,26]$ demonstrated that failing human myocardium had low level of $\mathrm{Na}^{+}-\mathrm{K}^{+}$-ATPase concentration and, a consequence, had elevated intracellular $\mathrm{Na}^{+}$levels $[12,13]$. Moreover, studies in rodent hearts showed that decrease in ATP production effects $\mathrm{Na}^{+}-\mathrm{K}^{+}$-ATPase activity and leads to elevated intracellular $\mathrm{Na}^{+}$concentration [27].

Involvement of $\mathrm{Na}^{+}-\mathrm{K}^{+}$-ATPase in electrolyte homeostasis makes ATP12A a possible candidate gene that might modify cardiac function. In our study, we investigated the genetic variation which is located in a transcription factor binding site of the ATP12A promoter area. Several transcription factors can bind this promoter region including CTCF (CCCTC-binding factor) and NF-kB that both act in NF-kB cascade [19]. Of notice, levels of NF- $k B$ were increased in failing human myocardium [28]. Therefore, in conditions related to disturbances in ATP production such as ischemia or heart failure, these transcription factors might modify expression of $\mathrm{Na}^{+}-\mathrm{K}^{+}$-ATPase.

In our study we observed that subjects with minor $\mathrm{C}$ allele in the ATP12A promoter area had significantly higher transmitral and tissue Doppler velocities during early diastole and, therefore, demonstrated an enhanced early myocardial relaxation compared to overall population mean. Overall, these findings might be indicative of the functional importance of the described genetic variation in ATP12A. However, we do not know whether rs10507337 $\mathrm{C}$ allele is associated with increased or decreased expression of the ATP12A gene. The precise mechanisms underlying the association of LV diastolic function with ATP12A remain to be elucidated. 
Our echocardiographic estimation of diastolic function included measurement of diastolic blood flow (A, E) and mitral annular movement (a', e'), and calculation of several ratios $\left(E / A, e^{\prime} / a^{\prime}, E / e^{\prime}\right)$. Since each of these Doppler indexes represents a somewhat distinct feature of diastolic function, none of them is sufficient to stand alone [1]. On the other hand, because diastolic Doppler indexes are measuring the same construct, e.g. velocities of transmitral blood flow and LV mitral annulus movement during diastole and its ratios, it is possible to reduce the measured phenotypes into a principal component (composite score) that will account for most of the variance in the variables. Indeed, all used diastolic indexes are intra-correlated and, therefore, some redundancy is expected. This continuously and normally distributed diastolic Doppler score accounted for $61.5 \%$ of the variance in the contributing indexes. In our study, subjects with better diastolic function had higher values of the composite score. Similarly, the composite diastolic score was significantly higher in rs10507337 C allele carriers and in CCTTC haplotype carriers. Because of the linkage disequilibrium between rs10507337 and rs12872010, this association was also observed in rs12872010 minor allele carriers. Hence, our findings remained consistent and we may conclude that the composite score of diastolic Doppler velocities might be used in genomic studies as a phenotype describing LV diastolic function.

The present study must be interpreted within the context of its limitations and strengths. All participants were white Europeans. Thus, the association cannot be generalized to other ethnic or racial groups. Transmitral and TDI diastolic velocities are quantitative echocardiographic traits that arise from complex interaction between multiple genes, hemodynamic, and environmental factors and are prone to measurement error. For the present study, one experienced observer in each center performed all echocardiographic examinations. Digitally stored images were post-processed centrally by one observer with high reproducibility [4]. There was also a high degree of internal consistency between the results of the population-based and family-based analyses. The between-family components for rs10507337 were not statistically significant, which makes it unlikely that our results are driven by population stratification.

\section{Conclusion}

Left ventricular diastolic function as assessed by conventional and tissue Doppler indexes was associated with genetic variation in the ATP $12 A$ promoter. We observed that carriers of rs10507337 C allele or CCTTC haplotype had better pattern of myocardial relaxation as compared to non-carriers. Further studies are necessary to clarify the functional significance of this genetic variation.

\section{Additional file}

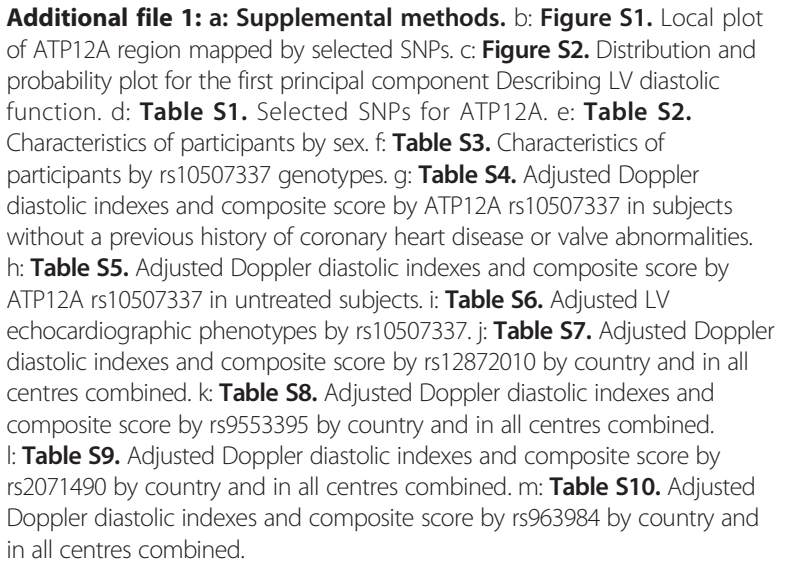

\section{Abbreviations}

A: the transmitral peak late diastolic velocity; a': the peak late diastolic mitral annular velocity; ATP: Adenosine triphosphate; ATP12A: Gene that encode the catalytic alpha subunit of $\mathrm{Na}^{+} / \mathrm{K}^{+}$-ATPase; BSA: Body surface area; E: the transmitral peak early diastolic velocity; E/A ratio: E velocity/A velocity ratio; $\mathrm{e}^{\prime}$ : the peak early diastolic mitral annular velocity; e'/a' ratio: e' velocity/a' velocity ratio; E/e' ratio: E velocity/e' velocity ratio; EPOGH: European Project on Genes in Hypertension; EF: Ejection fraction; FLEMENGHO: Flemish Study on Environment, Genes and Health Outcomes; LV: Left ventricle; MAF: Minor allele frequency; QTDT: Quantitative transmission disequilibrium test; SD: Standard deviation; TDI: Tissue Doppler Imaging.

\section{Competing interests}

The authors declare that they have no competing interests.

\section{Authors' contributions}

TK had full access to all of the data and takes responsibility for the integrity of the data and the accuracy of the statistical analysis. Study concept and design: JK, ES, JAS, TK. Acquisition of the phenotypic data: JK, VT, KSS, AR, MKK, SM, EC, DC, KKJ, TK. Molecular analysis: ES, DB, DC, TN. Statistical analysis and manuscript preparation: JK, ES, LT, TK. Critical revision of the manuscript for important intellectual content: VT, KSS, AR, DB, MKK, SM, EC, DC, KKJ, DC, TN, JAS. Obtained funding: SM, EC, DC, KKJ, DC, JAS, TK. All authors read and approved the final manuscript.

\section{Acknowledgments}

The European Union (grants IC15-CT98-0329-EPOGH, LSHM-CT-2006-037093InGenious HyperCare, HEALTH-F4-2007-201550-HyperGenes, HEALTH-2011278249-EU-MASCARA, HEALTH-F7-305507-HoMAGE, and ERC Advanced Grant-2011-294713-EPLORE) supported the Research Unit Hypertension and Cardiovascular Epidemiology (Leuven, Belgium). The Research Unit

Hypertension and Cardiovascular Epidemiology also received grants from the Fonds voor Wetenschappelijk Onderzoek Vlaanderen, Ministry of the Flemish Community, Brussels, Belgium (grants G.0734.09, G.0880.13 and G. 0881.13). The Department of Health, University of Milano and Genomics and Bioinformatics Platform, Fondazione Filarete are supported by InterOmics (PB05 MIUR-CNR Italian Flagship Project).

\section{Author details}

${ }^{1} \mathrm{KU}$ Leuven Department of Cardiovascular Sciences, Research Unit Hypertension and Cardiovascular Epidemiology, University of Leuven, Leuven, Belgium. ${ }^{2}$ Hypertension Division, Department of Internal Medicine, University Clinical Centre Ljubljana, Ljubljana, Slovenia. ${ }^{3}$ Department of Health, University of Milano and Genomics and Bioinformatics Platform, Fondazione Filarete, Milano, Italy. ${ }^{4}$ Department of Medicine, University of Padova, Padova, Italy. ${ }^{5} \mathrm{MRC}$ Unit for Lifelong Health and Ageing at University College London, London, UK. ${ }^{6}$ First Department of Cardiology, Interventional Electrocardiology and Hypertension, Jagiellonian University Medical College, Krakow, Poland. 'Institute of Internal and Preventive Medicine, Novosibirsk, 
Russian Federation. ${ }^{8}$ Novosibirsk State Medical University, Novosibirsk, Russian Federation. ${ }^{9}$ Department of Public Health, Occupational and Environmental Medicine, KU Leuven, Leuven, Belgium. ${ }^{10}$ Centre for Environmental Sciences, Hasselt University, Diepenbeek, Belgium. ${ }^{11}$ Department of Epidemiology, Maastricht University, Maastricht, Netherlands.

Received: 4 August 2014 Accepted: 22 October 2014

Published online: 04 November 2014

\section{References}

1. Kuznetsova T, Herbots L, Yin Y, Stolarz-Skrzypek K, Staessen J: Systolic and diastolic left ventricular dysfunction: from risk factors to overt heart failure. Expert Rev Cardiovasc Ther 2010, 8:251-258

2. Abhayaratna W, Marwick T, Smith W, Becker N: Characteristics of left ventricular diastolic dysfunction in the community: an echocardiographic survey. Heart 2006, 92:1259-1264.

3. Kloch-Badelek M, Kuznetsova T, Sakiewicz W, Tikhonoff V, Ryabikov A, González A, López B, Thijs L, Jin Y, Malyutina S, Stolarz-Skrzypek K, Casiglia E, Díez J, Narkiewicz K, Kawecka-Jaszcz K, Staessen JA: Prevalence of left ventricular diastolic dysfunction in European populations based on cross-validated diagnostic thresholds. Cardiovasc Ultrasound 2012, 10:10.

4. Kuznetsova T, Herbots L, López B, Jin Y, Richart T, Thijs L, González A, Herregods MC, Fagard RH, Díez J, Staessen JA: Prevalence of left ventricular diastolic dysfunction in a general population. Circ Heart Fail 2009, 2:105-112.

5. Redfield M, Jacobsen S, Burnett JJ, Mahoney D, Bailey K, Rodeheffer R: Burden of systolic and diastolic ventricular dysfunction in the community: appreciating the scope of the heart failure epidemic. JAMA 2003, 289:194-202

6. Nagueh SF, Appleton CP, Gillebert TC, Marino PN, Oh JK, Smiseth OA, Waggoner AD, Flachskampf FA, Pellikka PA, Evangelista A: Recommendations for the evaluation of left ventricular diastolic function by echocardiography. J Am Soc Echocardiogr 2009, 22:107-133.

7. Wang M, Yip GW, Wang AY, Zhang Y, Ho PY, Tse MK, Yu CM, Sanderson JE Tissue Doppler imaging provides incremental prognostic value in patients with systemic hypertension and left ventricular hypertrophy. J Hypertens 2005, 23:183-191.

8. Dokainish H, Zoghbi WA, Lakkis NM, Ambriz E, Patel R, Quinones MA Nagueh SF: Incremental predictive power of B-type natriuretic peptide and tissue Doppler echocardiography in the prognosis of patients with congestive heart failure. J Am Coll Cardiol 2005, 45:1223-1226.

9. Kuznetsova T, Thijs L, Knez J, Herbots L, Zhang Z, Staessen J: Prognostic value of left ventricular diastolic dysfunction in a general population. J Am Heart Assoc 2014, 3(3):e000789.

10. Kloch-Badelek M, Knez J, Tikhonoff V, Thijs L, Sakiewicz W, Ryabikov A, Stolarz-Skrzypek K, Jin Y, Malyutina S, Casiglia E, Narkiewicz K, Czarnecka D, Kawecka-Jaszcz K, Staessen JA, Kuznetsova T, European Project on Genes in Hypertension (EPOGH) Investigators: Heritability and other determinants of left ventricular diastolic function in the family-based population study. $J$ Hypertens 2014, 32:1854-1861.

11. Despa S, Bers $\mathrm{D}: \mathrm{Na}^{+}$transport in the normal and failing heart - remember the balance. J Mol Cell Cardiol 2013, 61:2-10.

12. Pieske $\mathrm{B}$, Houser $\mathrm{S}$ : $[\mathrm{Na}+] \mathrm{i}$ handling in the failing human heart. CardiovasC Res 2003, 57:874-886.

13. Pogwizd S, Sipido K, Verdonck F, Bers D: Intracellular Na in animal models of hypertrophy and heart failure: contractile function and arrhythmogenesis. Cardiovasc Res 2003, 57:887-896.

14. Pieske B, Maier L, Piacentino V, Weisser J, Hasenfuss G, Houser S: Rate dependence of $[\mathrm{Na}+]$ i and contractility in nonfailing and failing human myocardium. Circulation 2002, 106:447-453.

15. Blanco G, Mercer R: Isozymes of the Na-K-ATPase: heterogeneity in structure, diversity in function. Am J Physio/ 1998, 275:633-650.

16. Jaisser $\mathrm{F}$, Beggah $\mathrm{A}$ : The nongastric $\mathrm{H}+-\mathrm{K}+-\mathrm{ATPases}$ : molecular and functional properties. Am J Physiol 1999, 276:812-824.

17. Grishin A, Caplan M: ATP1AL1, a member of the non-gastric H, K-ATPase family, functions as a sodium pump. J Biol Chem 1998, 273:27772-27778.

18. Salvi E, Kutalik Z, Glorioso N, Benaglio P, Frau F, Kuznetsova T, Arima H, Hoggart C, Tichet J, Nikitin YP, Conti C, Seidlerova J, Tikhonoff V, StolarzSkrzypek K, Johnson T, Devos N, Zagato L, Guarrera S, Zaninello R, Calabria A, Stancanelli B, Troffa C, Thijs L, Rizzi F, Simonova G, Lupoli S, Argiolas G, Braga D, D'Alessio MC, Ortu MF, et al: Genomewide association study using a high-density single nucleotide polymorphism array and casecontrol design identifies a novel essential hypertension susceptibility locus in the promoter region of endothelial NO synthase. Hypertension 2012, 59:248-255.

19. Sverdlov V, Kostina M, Modyanov N: Genomic organization of the human ATP1AL1 gene encoding a ouabain-sensitive H, K-ATPase. Genomics 1996, 32:317-327.

20. Abecasis $G$, Cardon L, Cookson W: A general test of association for quantitative traits in nuclear families. Am J Hum Genet 2000, 66:279-292.

21. Kinoshita $\mathrm{K}$, Ashenagar M, Tabuchi M, Higashino $\mathrm{H}$ : Whole rat DNA array survey for candidate genes related to hypertension in kidneys from three spontaneously hypertensive rat substrains at two stages of age and with hypotensive induction caused by hydralazine hydrochloride. Exp Ther Med 2011, 2:201-212.

22. Geering K: The functional role of beta subunits in oligomeric P-type ATPases. J Bioenerg Biomembr 2001, 33:425-438.

23. Crambert G, Horisberger J, Modyanov N, Geering K: Human nongastric $\mathrm{H}+-\mathrm{K}+-$ ATPase: transport properties of ATP1al1 assembled with different beta-subunits. Am J Physiol Cell Physiol 2002, 283:305-314.

24. Modyanov N, Mathews P, Grishin A, Beguin P, Beggah AT, Rossier BC, Horisberger JD, Geering K: Human ATP1AL1 gene encodes a ouabainsensitive H-K-ATPase. Am J Physiol 1995, 269:992-997.

25. Schwinger R, Wang J, Frank K, Müller-Ehmsen J, Brixius K, McDonough AA, Erdmann E: Reduced sodium pump alpha1, alpha3, and beta1-isoform protein levels and $\mathrm{Na}+, \mathrm{K}+-$ ATPase activity but unchanged $\mathrm{Na}+-\mathrm{Ca} 2+$ exchanger protein levels in human heart failure. Circulation 1999, 99:2105-2112

26. Schwinger R, Bundgaard H, Muller-Ehmsen J, Kjeldsen K: The Na, K-ATPase in the failing human heart. Cardiovasc Res 2003, 57:913-920.

27. Jansen $M$, Shen $H$, Zhang L, Wolkowicz $P$, Balschi J: Energy requirements for the $\mathrm{Na}+$ gradient in the oxygenated isolated heart: effect of changing the free energy of ATP hydrolysis. Am J Physiol Heart Circ Physiol 2003, 285:2437-2445.

28. Wong S, Fukuchi M, Melnyk P, Rodger I, Giaid A: Induction of cyclooxygenase-2 and activation of nuclear factor-kB in myocardium of patients with congestive heart *failure. Circulation 1998, 98:100-103.

\section{doi:10.1186/s12881-014-0121-6}

Cite this article as: Knez et al: Left ventricular diastolic function associated with common genetic variation in ATP12A in a general population. BMC Medical Genetics 2014 15:121.

\section{Submit your next manuscript to BioMed Central and take full advantage of:}

- Convenient online submission

- Thorough peer review

- No space constraints or color figure charges

- Immediate publication on acceptance

- Inclusion in PubMed, CAS, Scopus and Google Scholar

- Research which is freely available for redistribution 